\title{
The Effect of Dopant Type on The Morphology and Electrical Properties of Hollow Polyester Fabrics
}

\author{
${ }^{1}$ Hidayet Köse and ${ }^{* 2}$ Suat Cetiner \\ ${ }^{1}$ University of KahramanmarasSutçu Imam, USKIM, Kahramanmaraş, Turkey \\ *2 University of KahramanmarasSutçu Imam, Department of Textile Engineering, Kahramanmaraş, Turkey
}

\begin{abstract}
:
Intrinsically conducting polymers (ICPs) have been intensively the subject of research since these polymers have superlative electrical and thermophysical properties. Due to the low hydrogen content and aromatic structure, they show perfect chemical, thermal, and oxidative stability and are practically insoluble in all common solvents. Also these polymers are latently electrical conducting materials, especially when doped. Polypyrole (PPy) is a very promising conducting polymer. It can be in easy way processes and has many interesting electrical properties. Also it is chemically and thermally stable. Like many other fully aromatic polymers, PPy is an electrical insulator, however, when oxidized it becomes an electrical conductor.The conductivity of PPy strongly consists in the preparation technique, and on the polymer additives and can be increased by about two orders of magnitude. In this study, electrically conductive hollow fabrics were prepared via in situ chemical polymerization method and scanning electron microscopy (SEM) and electrical properties of conductive hollow fabrics were invesigated.
\end{abstract}

Keywords:Conductivepolymer, Hollowfabrics, Polypyrrole

\section{Introduction}

Electrically conductive polyester composite textile would be very useful in various fields, including the manufacture of antistatic material, electromagnetic interference (EMI) shielding material,strain sensors, sensing elements, heating elements,sport garments,electrotherapy treatment, rehabilitation, motion capture device and packing of electronic components and equipment, etc.There have beensome publications in the area of conductive polymercoated textiles reporting chemical polymerisation inthe presence of the substrate, which was exposed to anoxidizing agent, monomer and dopant simultaneouslyor sequentially using specific mixtures, agitation timesand temperatures. Polymer conductivity depends onthe concentrations of the oxidant, monomer, dopant,polymerisation temperature and time [1]. The methods usedin chemical polymerizationused to producePPy-conducting textilesare as follows: in situ polymerization,two-step polymerization, emulsionpolymerization and vapor phase polymerization. The synthesis of PPy on textile substrates is carriedout by ferric chloride (FeCl3) and ammonium persulfate(APS). Sulfonic acid-based dopants such as anthraquinonesulfonic acid (AQSA), dodecylbenzenesulfonicacid sodium salt (DBSA), ptoluene sulfonic acid(PTSA), naphthalenedisulfonic acid (NDSA), benzenesulfonic acid (BSA) and naphthalenesulfonic acid(NSA) are used[2].

*Corresponding author: Address: Faculty of Engineering, Department of Textile Engineering Kahramanmaras Sütcü Imam University, 46050, Kahramanmaras TURKEY. E-mail address: suatcetiner@ksu.edu.tr, Phone: $\mathbf{+ 9 0 3 4 4 3 0 0 1 6 4 9}$ 
In recent years, conductive PET composite textile produced by in situ chemical polymerization of pyrrolehas aroused a great interest. The in situ polymerization method is a process that the monomer such as aniline and pyrrole is polymerized in the presence of PET fibers and deposited onto the surface of the PET fibers. In general, the conductive PPy composites are less stable in airdue to their reactivity with a variety of atmospheric chemicals, especially oxygen. Because of thelow oxidation potential of PPy, the redox reaction of PPy is moresensitive to the oxygen than those polymers that are more difficultto oxidize. The conductivity stability is one of important properties of conductive composites [3].

Different natural and synthetic fibres have been subjected to polymerisation of pyrrole (Py) and other conductive polymers for the production of conductive materials. A great deal of work has beencarried out on synthetic fibres, especially polyester, which takes the leading situation in the world fibre market in terms of fibre production and consumption. Comparatively less attention has been paid to other man-made fibres, such as polyamide or cellulose-based regenerated fibres, like viscose, cupro, and lyocell.As well as natural fibres are interested, cotton and,more recently, wool have concerned a great deal of attention[4].

In the present study, the PPy-coated hollow fabrics were produced in the cases of $\mathrm{FeCl}_{3}$ oxidant and $p$-TSA dopant by in situ oxidative polymerization. The electrical performances of the coated hollow fabrics were evaluated by electrical conductivity. Moreover, homogenity and morphology of the resulting polypyrrole layer were discussed. The thickness increases after polypyrrole deposition on the hollow fabrics were greater than $30 \%$ and polypyrrole deposited on the hollow fibers as a uniform film.

\section{Experimental}

\subsection{Materials}

A conductive monomer, Pyrrole, iron(III) chloride (FeCl3) and $p$-toluensulfonic acid monohydrate (p-TSA) were used without further purification. All reagents were supplied from Sigma-Aldrich. Acetone, ethanol and methanol were supplied from Merck. An undyed 100\% hollow fiber woven fabric with a $102 \mathrm{gr} / \mathrm{m}^{2}$ was woven.

\subsection{Method}

Method

Polypyrrole coated hollow fiber fabrics were produced by in-situ chemical oxidative polymerization method. The iron(III) chloride and $p$-toluensulfonic acid monohydrate were mixtured at room temperature with a magnetic stirrer for $1 / 2 \mathrm{~h}$ in a $50 \mathrm{~mL}$ of distilled water. The hollow fiber fabrics with the dimensions of $5 \mathrm{~cm} \times 5 \mathrm{~cm}$ were rinsed in acetone and distilled water to remove the contaminations of oil residues and was dried in an oven at $60^{\circ} \mathrm{C}$. Then hollow fiber fabrics fabrics were wetted in distilled water which contains dissolved of iron(III)chloride and p-toluensulfonic acid monohydrate at $25{ }^{\circ} \mathrm{C}$. The calculated amount of pyrrole was added drop by drop to the hollow fiber fabric solution and mixtured for 3 hours with a magnetic stirrer at room temperature.

At the end of the polymerization, the polypyrrole coated conductive hollow fabric was rinsed with methanol, ethanol and distilled water for 5 minutes to remove the unpolymerized particles. The test specimens were allowed to dry at room temperature for 24 hours. 


\subsection{Characterization ofPolypyrroleCoated Hollow Fabrics}

Electrical analysis of the polypyrrole coated hollow fiber composite fabrics fabric was carried out by four point probe dc electrical conductivity measurement technique. The morphology of the polypyrrolecoated hollow fiber composite fabrics was observed with a Carl Zeiss Evo LS10 scanning electron microscope.

\section{Results}

The SEM image in Fig 1 shows a section of a hollow fiber fabric uncoated and coated with 0.4 (v/v) \% PPy film.

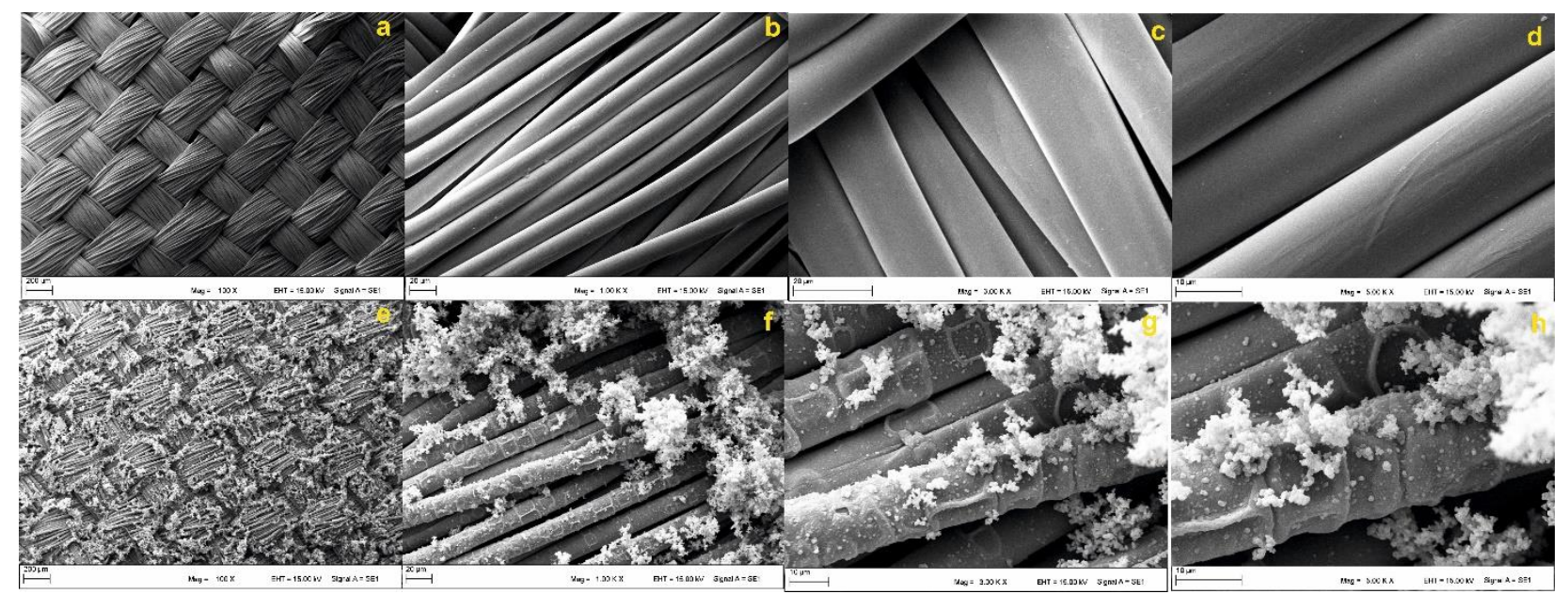

Figure 1. Surface Morphologies of Pristine hollow fiber fabric (a, b, c, d) and 0.4 (v/v) \% Polypyrrole coated hollow fiber fabric $(e, f, g, h)$

When the uncoated hollow fiber fabric had a quite smooth surface, a significant change on the surface of hollow fiber fabric was occurred after polypyrrole coating. The homogeneously distributed polypyrrole nanoparticle coatings were observed on the polypyrrole coated hollow fabric structures by scanning electron microscope.It can be clearly seen that there is a significant change on the surface of polypyrrole coated hollow fiber fabrics in the cases of PPy polymerized by $\mathrm{FeCl}_{3}$ and $p$-TSA. The clusters nanoparticles are observed on the surface of $\mathrm{F}\left(\mathrm{FeCl}_{3}+p\right.$ TSA), which results in a rough surface (Figures 1 (e-f-g-h). The coating appears quite smooth and there is a very homogeneous film-like dense layer.

The SEM image in Fig 2 shows a cross section of a hollow fiber fabric pristine and coated with $0.4(\mathrm{v} / \mathrm{v}) \%$ PPy film. 


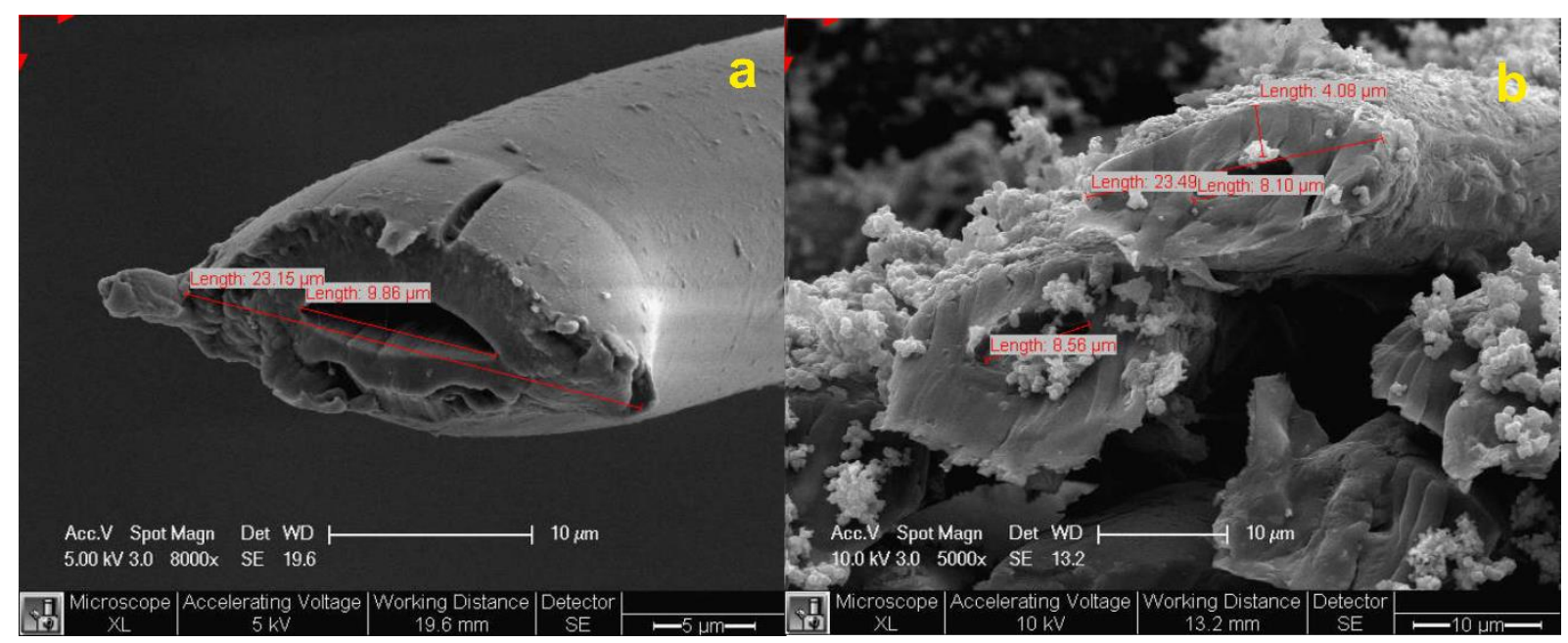

Figure 2. Cross Section Morphologies of Pristine hollow fiber fabric (a) and 0.4 (v/v) \% Polypyrrole coated hollow fiber fabric (b)

When the uncoated hollow fiber fabric had a quite smooth cross section, a significant change on the cross section of hollow fiber fabric was occurred after polypyrrole coating. The homogeneously distributed polypyrrole nanoparticle coatings were observed on cross section of the polypyrrole coated hollow fabric structures by scanning electron microscope (Figure 2)

\subsection{Electrical Properties of Conductive Hollow Fiber Fabric}

Figure 3 presents electrical behavior of polypyrrole coated hollow fiber fabric.

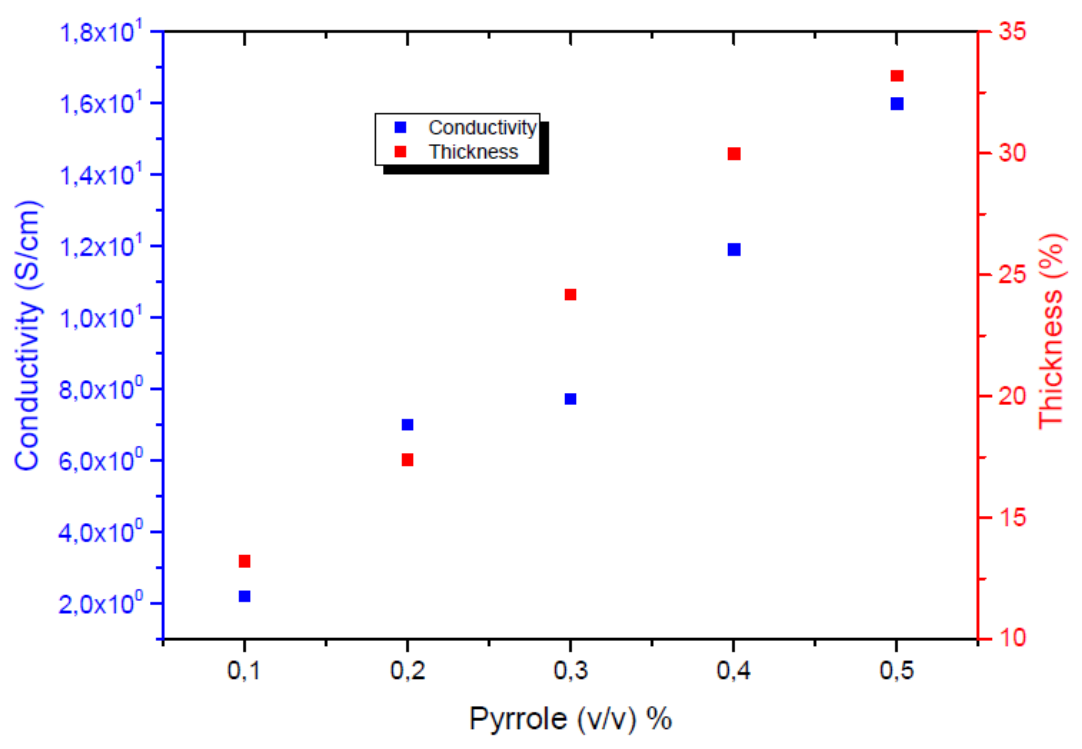

Figure 3. Metal-semiconductor behavior and deposition thickness of conductivehollow fiber fabric at different pyrrole concentrations

The conductivity and thickness of polypyrrole coated hollow fiber fabrics modified depending on the amount of pyrrole used in this study. That isthe conductivity and thickness increased when the amount of pyrrole increased (Figure 3). 


\subsection{Application of Conductive Hollow Fiber Fabric}

An house design with electrical circuit containing the treated hollow fiber fabric wasbuilt in order to test the accuracy of our approach of the highelectrically conductivity hollow fiber fabric method using the concentration 0,4 (v/v) \% Ppy as shown in Fig. 4, (which is showing the LEDs test).

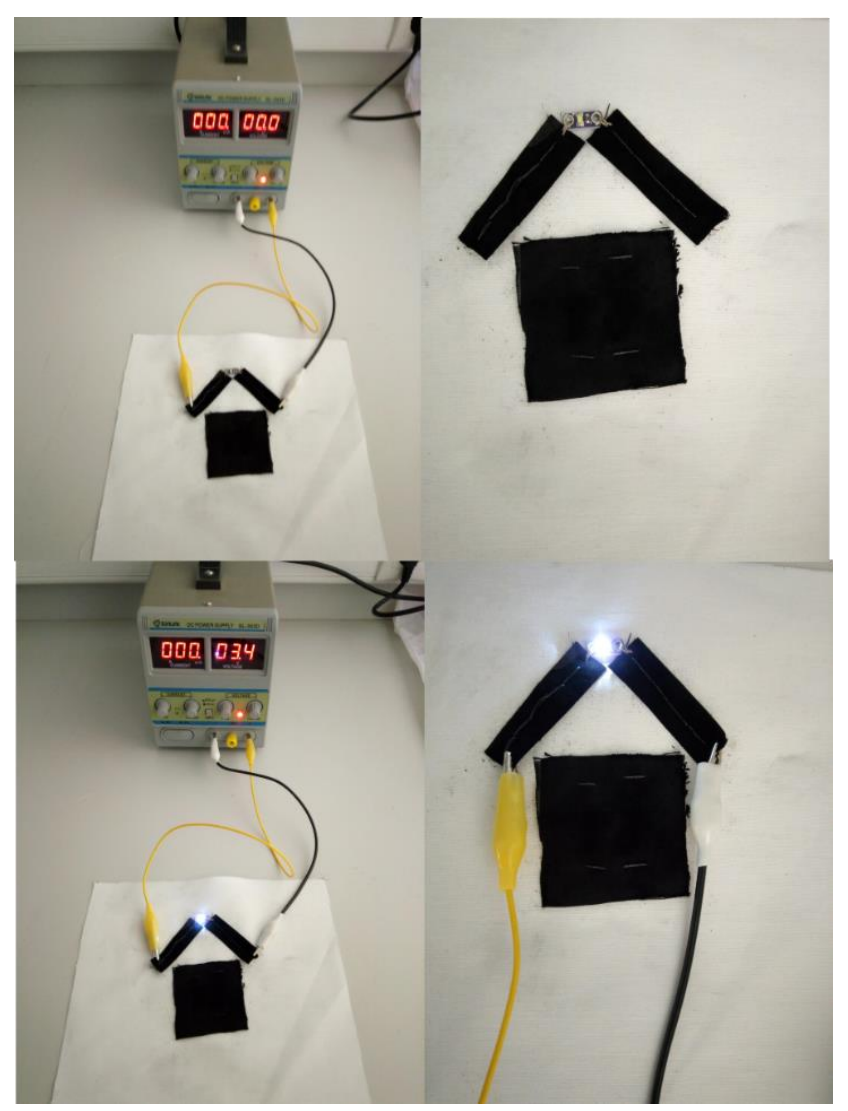

Figure 4. The inset is the conductive hollow fiber fabric device construction.

An DC potential of $3.4 \mathrm{~V}$ was then applied to the circuit through the power supply as shown in Fig. 4. The LEDs is powered to full intensity without any visible degradation occurring to the conductive hollow fiber fabric.

\section{Conclusions}

In conclusion, we have presented a method for the fabrication ofa highly electrically conductive hollow fiber fabric with metallic behavior,using the in situ chemical polymerization with pyrrole.

The conductive fabric constructed by this procedure could be useful in conducting wires, electrodes, and as functional fabrics in wearable electronics. The pyrrole-coated hollow fiber fabric exhibited metal-semiconductor behavior. 


\section{Acknowledgements}

This study was supported by the Scientific and Technological Research Council of Turkey (TUBITAK) under project code 217M743. The authors thank to Ahmet Munduz for the weaving process of multifilament hollow fibers.

\section{References}

[1]Kaynak, A., \& Beltran, R., Effect of synthesis parameters on the electrical conductivity of polypyrrole-coated poly(ethylene terephthalate) fabrics. Polymer International, 2003:52(6), 1021-1026.

[2]Cetiner, S., Dielectric and morphological studies of nanostructured polypyrrole-coated cotton fabrics. Textile Research Journal, 2014:84(14), 1463-1475.

[3] Li, J., Qian, X. R., Chen, J. H., Ding, C. Y., \& An, X. H., Conductivity decay of cellulosepolypyrrole conductive paper composite prepared by in situ polymerization method. Carbohydrate Polymers, 2010:82(2), 504-509.

[4] Cucchi, I., Boschi, A., Arosio, C., Bertini, F., Freddi, G., \& Catellani, M., Bio-based conductive composites: Preparation and properties of polypyrrole (PPy)-coated silk fabrics. Synthetic Metals, 2009:159(3-4), 246-253. 